\title{
Mod difference labeling of some classes of digraphs
}

\author{
B Sooryanarayana ${ }^{1 *}$ and Sunita Priya D Silva²
}

\begin{abstract}
A graph is a difference graph if there is a bijection $f$ from $V$ to a set of positive integers $S$ such that $x y \in E$ if and only if $|f(x)-f(y)| \in S$. A digraph $D=(V, E)$ is a mod difference digraph if there exist a positive integer $m$ and labeling $L: V \rightarrow\{1,2, \ldots, m-1\}$ such that $(x, y) \in E$ if and only if $L(y)-L(x) \equiv L(w)(\bmod m)$ for some $w \in V$. In this paper, we prove that the complete bipartite digraphs, oriented binary trees, ladder graphs and fan graphs are mod difference digraphs.
\end{abstract}

Keywords

Difference labeling, mod difference labeling, digraphs.

\section{AMS Subject Classification}

05C20, $05 \mathrm{C} 78$.

${ }^{1}$ Department of Mathematics, Dr. Ambedkar Institute of Technology, Bangalore-560056, India.

${ }^{2}$ Department of Mathematics, Sahyadri College of Engineering and Management, Mangalore-575009, India.

*Corresponding author: ${ }^{*} \mathrm{dr} \_b s n r a o @ d r-a i t . o r g ; ~{ }^{2}$ sunitapriya11@gmail.com

Article History: Received 17 July 2019; Accepted 12 December 2019

(C)2020 MJM

\section{Contents}

1 Introduction 32

2 Mod difference labeling of some classes of digraphs 32

3

Conclusion 35

References

\section{Introduction}

In this paper, we consider only finite simple graphs. Let $S$ be a finite multiset of real numbers, i.e. a finite collection of real numbers in which repetitions is permitted but order is irrelevant. The difference graph with signature $S$ is a finite digraph $\mathrm{G}$ with vertices labelled bijectively by $f$ from a vertex labelled $x$ to a vertex labelled $y$ exactly when $x-y \in S$. In [4] Harary introduced the concept of difference graphs similar to sum graphs and similar works we refer [8, 9]. Some classes of difference graphs (paths, trees, cycles, special wheels, complete graphs, complete bipartite graphs etc.) were investigated by Bloom, Burr, Eggleton, Gervacio, Hell and Taylor in the undirected $[2,3,12]$ as well as in the directed case [1].

The concept of mod difference digraph was introduced by S.M.Hegde and Vasudeva [6]. For the recent works we refer $[7,10,11]$.

Definition 1.1. A graph is a difference graph if there is a bijection $f$ from $V$ to a set of positive integers $S$ such that $x y \in E$ if and only if $|f(x)-f(y)| \in S$. A digraph $D=(V, E)$ is a mod difference digraph if there exist a positive integer $m$ and labeling $L: V \rightarrow\{1,2, \ldots, m-1\}$ such that $(x, y) \in E$ if and only if $L(y)-L(x) \equiv L(w)(\bmod m)$ for some $w \in V$.

They have shown some of the structural properties of mod difference digraphs in [5]. It is also proved that complete symmetric digraphs, unipaths and unicycles are mod difference digraphs [6].

\section{Mod difference labeling of some classes of digraphs}

In this section we present some results on mod difference labeling of some classes of digraphs.

Lemma 2.1. If $S$ is a proper signature of $\overleftrightarrow{K}_{n, n}$ then $S$ is partitioned into two disjoint sets $V_{1}$ and $V_{2}$ as follows:

$$
\begin{aligned}
& V_{1}=\left\{v_{i}=3 i-2,1 \leq i \leq n\right\} \\
& V_{2}=\left\{v_{i}=3 i-1,1 \leq i \leq n\right\}
\end{aligned}
$$

Proof. Consider an edge $x=u v$ in $\overleftrightarrow{K}_{n, n}$. Assume that both $u, v \in V_{1}$ (or $V_{2}$.) Without the loss of generality, let $u, v \in V_{1}$ then $u-v \in S$ for some $1 \leq i_{1}, i_{2} \leq n$ i.e $\left(3 i_{1}-2\right)-\left(3 i_{2}-2\right) \in$ $S$, for some $1 \leq i_{1}, i_{2} \leq n$ i.e $3\left(i_{1}-i_{2}\right) \in S$, but $3\left(i_{1}-i_{2}\right) \equiv$ $0(\bmod 3)$, a contradiction. Hence $V_{1}$ and $V_{2}$ form the partition of $\overleftrightarrow{K}_{n, n}$. 
Lemma 2.2. Let $V_{1}$ and $V_{2}$ be the bipartite vertex sets of $\overleftrightarrow{K}_{n, n}$ as defined in the above lemma. If $v_{i} \in V_{1}$ and $v_{j} \in V_{2}$ with $v_{i}<v_{j}, 1 \leq i, j \leq n$ then $v_{i}-v_{j} \in V_{1}$.

Proof. If $x=v_{i} v_{j}$ with $v_{i}<v_{j}$ is an edge in $\overleftrightarrow{K}_{n, n}$, then by Lemma $2.1, v_{i}=3 k_{1}-2$ and $v_{j}=3 k_{2}-1$, for some $1 \leq$ $k_{1}, k_{2} \leq n$. Therefore, $v_{j}-v_{i}=\left(3 k_{2}-1\right)-\left(3 k_{1}-2\right)=3\left(k_{2}-\right.$ $\left.k_{1}\right)+1$. Hence $0 \leq k_{1}, k_{2} \leq(n-1)$.

Taking,

$$
\begin{aligned}
k_{2}-k_{1} & =0, \text { we get, } v_{j}-v_{i}=1=3(1)-2 \in V_{1} \\
k_{2}-k_{1} & =1, \text { we get, } v_{j}-v_{i}=4=3(2)-2 \in V_{1} \\
& \vdots \\
k_{2}-k_{1} & =n-1, \text { we get, } v_{j}-v_{i}=1=3(n-1)+1 \in V_{1}
\end{aligned}
$$

Hence the proof.

Theorem 2.3. If the labeling $L$ given by $L\left(v_{i}\right)=a_{i}, \forall i, i=$ $1,2, \ldots, 2 n$ is a mod difference labeling of $\overleftrightarrow{K}_{n, n}$, then there exits an integer a such that $a_{i}=\left(3 n_{i}-k\right)$ a for $k=2, n_{i}=\frac{i+1}{2}$, when $i$ is odd and $k=1, n_{i}=\frac{i}{2}$, when $i$ is even and $m=3 n a$.

Proof. Without the loss of generality we take $a_{1}<a_{2}<\cdots<$ $a_{2 n}$. Set $a_{1}=a=(3(1)-2) a$. Since $L$ is a $\bmod$ difference labeling, consider $a_{2}-a_{1}=a_{l}$ for some $l$. Since $a_{1}<a_{2}$,we must have $a_{l}=a_{1}$. Then $a_{2}=a_{1}+a_{1}=2 a=(3(1)-1) a$. Consider $a_{3}-a_{2}=a_{m}$ for some m. Again $a_{2}<a_{3}$, we must have $a_{m}=a_{1}$ or $a_{2}$. If $a_{m}=a_{1}$, then $a_{3}=a_{1}+a_{2} \Rightarrow a_{3}-a_{1}=$ $a_{2} \Rightarrow$ there is an edge $a_{1} a_{3}$ in $\overleftrightarrow{K}_{n, n}$, a contradiction to the Lemma 2.1. Therefore, $a_{m}=a_{2}$ and hence $a_{3}=a_{2}+a_{2}=$ $4 a=[3(2)-2] a$.

We have proved the theorem is true for $n=1,2,3$. We assume the theorem is true for all $n<k$. Without the loss of generality consider $k$ to be even. Consider $a_{k}-a_{l}=a_{j}$ for some $j$, with $a_{l}<a_{k}$. Also $a_{j}<a_{k}$ We have observed that $a_{j}=a_{k-l} \in V_{1}$.

If $k$ is even ,then $l$ is odd. By Lemma 2.2, $j$ is also odd. By induction assumption, we have $a_{l}=\left[3 l_{i}-2\right] a$ and $a_{j}=$ $\left[3 j_{i}-2\right] a$, for some $1 \leq l_{i}, j_{i} \leq k$. Therefore,

$$
\begin{aligned}
a_{k} & =\left[3 j_{i}-2\right] a+\left[3 l_{i}-2\right] a \\
& =\left[3\left[\frac{j+1}{2}\right]-2\right] a+\left[3\left[\frac{l+1}{2}\right]-2\right] a \\
& =\left\{3\left[\frac{j+l+2}{2}\right]-4\right\} a \\
& =3\left\{\left[\frac{k-l+l+2}{2}\right]-4\right\} a \\
& =3\left\{\left[\frac{k+2}{2}\right]-4\right\} a \\
& =3\left\{\left[\frac{k}{2}\right]-1\right\} a \\
& =\left[3 k_{i}-1\right] a
\end{aligned}
$$

Therefore, the result is true for $k$. Hence by induction it is true for all positive $n$.
Also consider $a_{1}-a_{2 n} \equiv a_{j}(\bmod \mathrm{m})$ for some $\mathrm{j}$, since $a_{1} \in V_{1}$ and $a_{2 n} \in V_{2}$. Hence

$$
m=\left[(3 n-1)-1+3\left(\frac{j}{2}\right)-1\right] a=\left[3 n-3+3\left(\frac{j}{2}\right)\right] a .
$$

In the above $j \neq 2 n-1$, and $1 \leq j \leq n$. If $j>2$, then $m=$ $3(n+1) a$, which contradicts labeling condition. Therefore, $j=2$ which implies $m=3 n a$. Hence the proof.

Corollary 2.4. Any complete bipartite digraph $\overleftrightarrow{K}_{n, n}$ is a mod difference digraph.

Proof. Label the vertices $v_{1}, v_{2}, \ldots, v_{2 n}$ of $\overleftrightarrow{k}_{n, n}$, using the labeling $f\left(v_{i}\right)=i a$, for $1 \leq i \leq 3 n-1$, with $i \not \equiv 0(\bmod 3)$ and $a$ is a positive integer. We prove that $f$ is mod difference labeling of $\overleftrightarrow{K}_{n, n}$ with $m=3 n a$. Now, for all $i, j, i \neq j, i a-j a \equiv k a$ $(\bmod m)$, where

$$
k= \begin{cases}i-j, & \text { if } \quad i>j \\ 3 n+(i-j), & \text { if } \quad i<j .\end{cases}
$$

In both the cases $k \leq 2 n$. Therefore, there exits $k \leq 2 n$ such that $f\left(v_{i}\right)-f\left(v_{j}\right) \equiv f\left(v_{k}\right)(\bmod m)$, for all $i, j$ with $i \neq j$. Hence $f$ is a mod difference digraph.

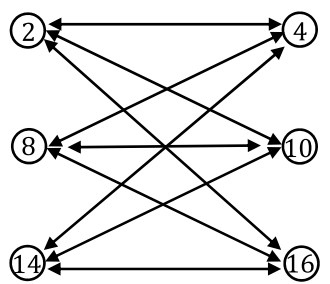

Figure 1. A mod difference labeling of the bi-directional digraph of $K_{3,3}$ with $m=18$ and $a=2$.

Definition 2.5. An $m$-ary tree $(m \geq 2)$ is a rooted tree in which each vertex has less than or equal to m children. When $m=2$, the rooted tree is called the binary tree.

Definition 2.6. An oriented binary tree $\vec{T}_{n}$ with $n$ vertices is said to be inspoken if all the parents in each level has indegree two.

Theorem 2.7. A oriented binary tree $\vec{T}_{n}$ whose internal vertices have indegree 2 and outdegree 1 is a mod difference digraph.

Proof. Let $\overrightarrow{T_{n}}=(V, E)$ be the oriented binary tree and $v$ be the root vertex. Let $v_{1}, v_{2}, \ldots, v_{p}$ be the end vertices in $\vec{T}_{n}$. First label all the $p$ leaves $h$ as $l\left(v_{1}\right)=a$ and $l\left(v_{i}\right)=2 l\left(v_{i-1}\right)+1$, $2 \leq i \leq p$. Then label all the internal vertices in the $h-1$ level by adding the labels of its children. Continuing the same procedure of labeling to all the non-pendent vertices in the previous level and so on., we finally reach the root $v$.

Now, for the vertices $u, v$ in $\vec{T}$ with $l(u>l(v)$, it is easy to observe that if $u v \in E$ with $l(u)>l(v)$, then $u$ is a parent 
of $v$, so $l(u)=l(v)+l(w)$ for some child $w$ of $u$. Hence $l(u)-l(v) \equiv l(w)$ whenever $u v \in E$.

Further, the label of $i^{t h}$ pendent vertex $v_{i}$ is $l\left(v_{i}\right)=2^{i}(a+$ 1) -1 and the level of the internal vertices is $l\left(u_{i}\right)=\left(2^{i_{1}}+\right.$ $\left.2^{i_{2}}+\cdots+2^{i_{2^{k}}}\right)(a+1)-2^{k}$ for some $k \in Z^{+}$.

if $u_{i}$ and $u_{j}$ be any two non-adjacent vertices of $\vec{T}$, then we have the following cases

CASE 1: $u_{i}$ and $u_{j}$ are pendent vertices

In this case $l\left(u_{i}\right)-l\left(u_{j}\right)=l\left(v_{i}\right)-l\left(v_{j}\right)=\left[2^{i}(a+1)-1\right]-$ $\left[2^{j}(a+1)-1\right]=\left(2^{i}-2^{j}\right)(a+1) \notin l(S) \Rightarrow u_{i} u_{j} \notin E$.

CASE 2: $u_{i}$ is a pendent and $u_{j}$ is an internal vertex.

In this case $l\left(u_{j}\right)-l\left(u_{i}\right)=\left(2^{i_{1}}+2^{i_{2}}+\cdots+2^{i_{2}}\right)(a+1)-$ $2^{k}-\left(2^{i}\right)(a+1)+1=\left(2^{i_{1}}+2^{i_{2}}+\cdots+2^{i_{2}}+2^{i}\right)(a+1)-$ $\left(2^{k}-1\right) \notin l(S) \Rightarrow u_{i} u_{j} \notin E$

CASE 3: $u_{i}$ and $u_{j}$ are internal vertices

In this case $l\left(u_{j}\right)-l\left(u_{i}\right)=\left(2^{j_{1}}+2^{j_{2}}+\cdots+2^{j_{2}}\right)(a+1)-$ $2^{k}-\left(2^{i_{1}}+2^{i_{2}}+\cdots+2^{i_{2} l}\right)(a+1)-2^{l}=\left(2^{j_{1}}+2^{j_{2}}+\cdots+\right.$ $\left.2^{j_{2}}-2^{i_{1}}-2^{i_{2}}-\cdots-2^{i_{2} l}\right)(a+1)-\left(2^{k}+2^{l}\right) \notin l(S) \Rightarrow u_{i} u_{j} \notin$ $E$ whenever $k \neq l$ (i.e $u_{i} \neq u_{j}$ ).

Hence $\vec{T}$ is a mod difference digraph. $\overrightarrow{T_{7}}$

A mod difference digraph of complete oriented binary tree

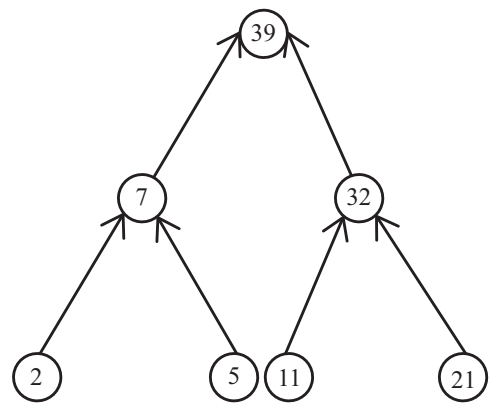

Figure 2. A binary tree with $a=2$ and $m=40$.

Definition 2.8. A fan graph $F_{m, n}$ is defined as the graph join $\overline{K_{m}}+P_{n}$, where $\bar{K}$ is the empty graph on $m$ vertices and $P_{n}$ is the path graph of $n$ vertices. The case $m=1$,corresponds to the usual fan graphs, while $m=2$ corresponds to the double fan graphs etc.

Definition 2.9. An oriented fan graph $\overrightarrow{F_{1, n}}$ is said to be an unipath fan, if the path of the fan is unidirectional.

Definition 2.10. An oriented fan graph $\overrightarrow{F_{1, n}}$ with $n+1$ vertices is called outspoken(inspoken), if indegree (outdegree) of the apex vertex is 0 .

Theorem 2.11. An unipath fan $\overrightarrow{F_{1, n}}$ with $n+1$ vertices is mod difference digraph, if indegree of the apex vertex is one.

Proof. Let $\overrightarrow{F_{1, n}}=(V, E)$ be an unipath fan. Let $V=\left\{v_{0}, v_{1}, v_{2}\right.$, $\left.\ldots, v_{n}\right\}$ and $E=\left\{v_{0} v_{i}: 1 \leq i \leq n\right\} \bigcup\left\{v_{i} v_{i+1}: 1 \leq i \leq n-1\right\}$, where $v_{0}$ is the apex vertex.
Label the apex vertex $v_{0}$ by 2 and label each vertex $v_{i}$ of the path by $2 i-1, i=1,2, \ldots, n$. With modular value $m=$ $2(n+1)$, this labeling scheme generates the signature for $\overrightarrow{F_{1, n}}$.

For $v_{i}-v_{0}=(2 i-1)-2=2(i-1)-1=v_{i-1}$, for all $i=2,3,4, \ldots, n$.

For $i=1$, we have $v_{0}-v_{1}=2-1=1 \in V$.

Also for $1 \leq i, j \leq n, i=j+1$,

$$
v_{i}-v_{j}=(2 i-1)-(2 j-1)=2(i-j)=2=v_{0} .
$$

Since for every $i, j \in\{1,2, \ldots, n\}$, we have $v_{i}-v_{j}$ is an even number greater than 2 and is not in $V$ under modulo $m$. Hence the labeling does not induces any additional edges. Hence $\overrightarrow{F_{1, n}}$ is mod difference digraph.

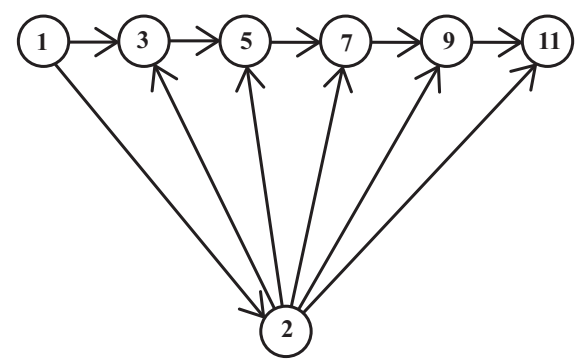

Figure 3. A mod difference labeling of an Unipath Fan with $m=14$.

If the signature of the mod difference digraph contains 0 integer, then such a digraph is named as $\bmod ^{*}$ difference digraph. S.M Hegde and Vasudeva [5] introduced $\bmod ^{*}$ difference digraph and defined as follows:

Definition 2.12. A simple digraph $D$ is called a mod $^{*}$ difference digraph if there exists a positive integer $m$ and a labeling $f$ of the vertices of $D$ with distinct elements of $f=$ $\{0,1,2, \ldots, m-1\}$ such that for the vertices $u$ and $v$ there exists an arc from $u$ to $v$ (denoted as $u \rightarrow v$ ) if and only if there is a vertex $w$ such that $(f(v)-f(u)) \equiv f(w)(\operatorname{modm})$. The function $f$ is called a mod $^{*}$ difference labeling of digraph $D$.

Theorem 2.13. An unipath outspoken fan $\overrightarrow{F_{1, n}}$ with $n+1$ vertices is a mod $^{*}$ difference digraph.

Proof. Consider the signature $S=\left\{0,1,2, \ldots, 2^{n-1}\right\}$ and modular value $m=2^{n}$. We prove that if $\mathrm{S}$ is the signature of an unipath outspoken fan. Let $\left\{v_{0}, v_{1}, \ldots, v_{n}\right\}$ be the vertices of unipath outspoken fan where $v_{0}$ is the apex vertex.

Let $v_{0}=0, v_{i}=2^{i-1}$ for $i=1,2,3, \ldots, n$. Now, $v_{i}-v_{0}=v_{i}$ for $i=1,2, \ldots, n$. For the vertices on the path and $i \neq j$, we have $v_{i}-v_{j}=2^{i-1}-2^{j-1} \in S$ if and only if $i=j+1$. Hence the proof.

Definition 2.14. An oriented Ladder graph $\overrightarrow{L_{n}}=\overrightarrow{P_{n}} \times \overrightarrow{P_{2}}$ is said to be oriented unipath ladder if the path $P_{n}$ is unidirectional. 


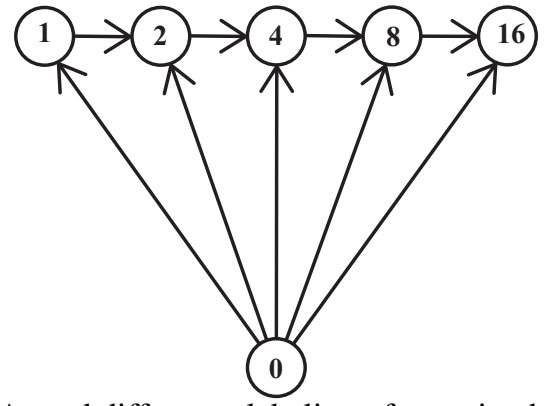

Figure 4. A mod difference labeling of an unipath outspoken Fan with $m=32$.

Theorem 2.15. An oriented unipath Ladder $\overrightarrow{L_{n}}$ is a mod difference digraph.

Proof. Let $\overrightarrow{L_{n}}=\overrightarrow{P_{n}} \times \overrightarrow{P_{2}}$ be an oriented unipath ladder. We label the vertices of $\overrightarrow{L_{n}}$ as $v_{1}, v_{2}, \ldots, v_{2 n}$. A ladder contains two paths, one path contains the vertices of the form $v_{2 i-1}$, $1 \leq i \leq n-1$ and the other path contains the vertices of the form $v_{2 i}, 1 \leq i \leq n$. The edge set contains the edges of the form :

$\left\{v_{2 i} v_{2 i+2}, v_{2 i-1} v_{2 i+1}, v_{2 i} v_{2 i-1}, 1 \leq i \leq n\right\}$.

We define the labeling function $f$ as:

$$
\begin{aligned}
f\left(v_{2 i}\right) & =2^{i}, 1 \leq i \leq n \\
f\left(v_{2 i-1}\right) & =2^{i+1}-3,1 \leq i \leq(n-1)
\end{aligned}
$$

We prove that $f$ is a mod difference labeling of $\overrightarrow{L_{n}}$ with $m=$ $2\left(2^{n+1}-3\right)$. That is, we show that $f\left(v_{i}\right)-f\left(v_{j}\right) \equiv f\left(v_{k}\right)$ (mod $m$ ), for some $k, 1 \leq k \leq 2 n$ if and only if $v_{j} v_{i}$ is an edge in $\overrightarrow{L_{n}}$.

For an edge $v_{2 i} v_{2 i+2}, 1 \leq i \leq(n-1)$, we have,

$$
\begin{aligned}
f\left(v_{2 i+2}\right)-f\left(v_{2 i}\right) & =2^{i+1}-2^{i} \\
& =2^{i}(2-1) \\
& =2^{i} \\
& =f\left(v_{2 i}\right)(\bmod m)
\end{aligned}
$$

For an edge $v_{2 i-1} v_{2 i+1}, 1 \leq i \leq(n-1)$ we have,

$$
\begin{aligned}
f\left(v_{2 i+1}\right)-f\left(v_{2 i-1}\right) & =\left[2^{(i+1)+1}-3\right]-\left[2^{i+1}-3\right] \\
& =2.2^{i+1}-2^{i+1} \\
& =2^{i+1} \\
& =f\left(v_{2(i+1)}\right)(\bmod m)
\end{aligned}
$$

For an edge $v_{2 i} v_{2 i-1}$, for $i>1$, we have,

$$
\begin{aligned}
f\left(v_{2 i}\right)-f\left(v_{2 i-1}\right) & =2^{i}-\left[2^{i+1}-3\right] \\
& =2.2^{i}-2^{i}-3 \\
& =2^{i}-3 \\
& =f\left(v_{2(i-1)-1}\right) \\
& =f\left(v_{2 i-3}\right)(\bmod m)
\end{aligned}
$$

Finally, for $\mathrm{i}=1, f\left(v_{2}\right)-f\left(v_{1}\right)=2-1=1(\bmod m)$. Hence $\overrightarrow{L_{n}}$ is a mod difference graph.

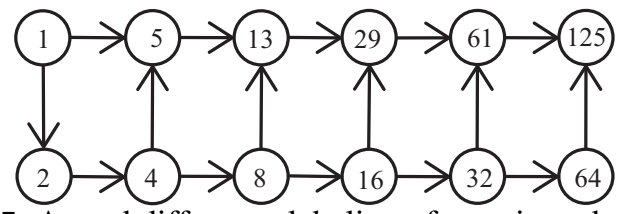

Figure 5. A mod difference labeling of an oriented unipath Ladder with $m=251$.

\section{Conclusion}

Cayley graphs are known to be excellent model for interconnection network due to their various properties like vertex transitivity, regularity, connectivity etc., Cayley graph $\operatorname{Cay}_{g}(A, S)$ is connected if and only if $S$ generates the group $A$. In particular, if $S=A-\{e\}$, where $e$ is the identity of $A$, then $\operatorname{Cay}(A, S)$ turns out to be a complete graph, which is a mod difference graph whenever the group operation is the usual addition. The Subgraph of a Cayley graph induced by $S$ is a problem of our interest. A graph $G$ that is a mod difference graph is a subgraph of certain Cayley graphs of a group of superset of $V(G)$.

The investigations made in this paper may enlighten a new direction for further development of a good interconnection networks which are subgraphs of Cayley Networks. The links are specific and can be identified as difference of the addresses. So, one can easily develop a routing algorithm to communicate between two nodes with the list of addresses of the nodes. .

\section{Acknowledgment}

Authors are very much thankful to the learned referees for their suggestions that helped to improve the paper.

\section{References}

[1] R.B. Eggleton and S.V. Gervacio, Some properties of difference graphs,Ars Combinatoria , 19(A)(1985), 113128.

[2] S. V. Gervacio, Which wheels are proper autographs?, Sea Bull. Math., 7 (1983), 41-50.

[3] G.S. Bloom, P.Hell and H. Taylor, Collecting autographs: $n$-node that have $n$-integers, Annals of New York Academy of Sciences, 314 (1979), 93-102.

[4] F. Harary, Sum graphs and difference graphs, Congressus Numerantium, 72 (1990), 101-108.

[5] S. M. Hegde and Vasudeva, Some structural properties of mod difference digraphs, (to appear).

[6] S. M. Hegde and Vasudeva, On mod difference labelings of digraphs, AKCE J.Graphs Combin., 6(2009), 79-84.

${ }^{[7]}$ H.Wang, B.Yao, and M.yao, Gernalized edge-magic total labellings of models from researching networks, Inform. Sci., 279(2014), 460-467.

[8] B. Sooryanarayana Jayalakshmi M and P Devadas Rao, Outer mod sum labelings of a graph, Internatial Journal 
of Information Science and Computer Mathematics, 2(2) (2010), 87-102.

[9] K. N. Meera and B. Sooryanarayana, Optimal outer sum number of a graphs, International Journal of Combinatorial Graph Theory and Applications , 2(4)(2011), , 23-35.

[10] L. L. Fontanil R. and G. Panopio, Independent set and vertex covering in a proper monograph determined through a signature,Australasian Journal of Combinatorics , 59(1)(2014), 64-71.

[11] M.A Seoud and E.F.Helmi, On difference graphs, 76(2016), 189-199.

[12] S.V.Gervacio, Difference graphs, Proceedings of the Second Franco-Southeast Asian Mathematics Conferences, University of Philippines, 1982.

$\operatorname{ISSN}(\mathrm{P}): 2319-3786$

Malaya Journal of Matematik

$\operatorname{ISSN}(\mathrm{O}): 2321-5666$

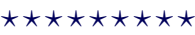

